\section{LA EVANESCENCIA DEL PUEBLO Y LOS USOS DEL ROMANCERO COMO FACTOR DE IDENTIDAD POLÍTICA}

\author{
Luis Díaz Viana \\ Instituto de Lengua, Literatura y Antropología (ILLA) \\ Centro de Ciencias Humanas y Sociales (CCHS) \\ Consejo Superior de Investigaciones Científicas. CSIC \\ luis.diaz@cchs.csic.es
}

\begin{abstract}
The compilation and study of the ballad tradition in general and the romancero in particular, have constituted, since they began to wake up the interest of the romantic folklorists, a specially propitious field to be used like factor of reconstruction of political identities. The fact that music and text they are in all ballad traditions united and combined fosters that potentiality. What this work discusses is why in the case of romancero the dominant tendency in research has been characterized until present times by the approach on a national or Hispanic scale, getting to even advise against the practice of the regional or provincial collections. And what methodological changes have been carried out by this position, which cannot be separated, on the other hand, from a theoretical concept of folk as national folk and which determines, in parallel, the evanescence and liquation of located and particular people, those who not only transmit, but also create culture, the true folk.
\end{abstract}

KEY WORDS: Ballad; music and literature; popular culture; folklore; anthropology; identity; politics.

Samuel G. Armistead, autor prestigioso y probablemente la mayor autoridad viva en materia romancística, ha insistido en alguna publicación reciente en ese interés añadido que para él tendrian, por el "conservadurismo característico" de los materiales encontrados, las compilaciones sobre las "áreas laterales más arcaizantes" de la romancística tradicional, pues aparecen -con frecuencia- en ellas verdaderas reliquias del más antiguo venero; a propósito de tales descubrimientos, escribe el propio Armistead que "el romance, de por sí solo, reivindica, sin lugar a la menor duda, el alto valor de una tradición oral capaz de mantener intacta su historicidad a lo largo de cinco largos siglos de tradición oral indocumentada" (Armistead, 2009, 11). Y saluda el mismo investigador, con entusiasmo, el "valor incalculable" que -en este sentido- las nuevas obras de

\section{THE EVANESCENCE OF PEOPLE, AND THE USES OF THE BALLAD TRADITION AS A FEATURE OF POLITICAL IDENTITY}

RESUMEN: La recopilación y el estudio de la balada en general, y del romancero en particular, ha constituido, desde que se empieza a despertar el interés de los folkloristas románticos, un campo especialmente propicio para ser utilizado como factor de reconstrucción de identidades políticas. El hecho de que música y texto estén en toda baladística inseparablemente unidos favorece esa potencialidad. Lo que este trabajo se plantea es por qué en el caso del romancero la tendencia dominante en su estudio se ha caracterizado hasta el presente por un enfoque a escala nacional o hispánico, llegando a desaconsejarse incluso la práctica de las colecciones regionales o provinciales. Y qué condicionamientos metodológicos ha acarreado esta postura, a la que no se puede desligar -de otro lado- de una determinada concepción teórica de pueblo, como pueblo-nación, la cual viene a determinar - paralelamente- la evanescencia y licuación de una gente o pueblo más localizados y concretos: quienes no sólo transmiten, sino también crean la cultura, el verdadero folk.

PALABRAS CLAVE: Romancero; literatura y música; cultura popular; folklore; antropologia; identidad; política.

recopilación sobre regiones o provincias no bien conocidas en lo romancístico podrian llegar a adquirir con el tiempo (Armistead, 2009, 13).

Pero, precisamente a tenor de una declaración como ésta y de otras que parecerian ir en dirección contraria, quizá convenga matizar algunas de las apreciaciones que suelen hacerse sobre la pertinencia o no de las recopilaciones efectuadas desde una perspectiva regional o incluso regionalista. Como Menéndez Pidal ya decía ( $y$, desde luego, no le faltaba razón) en una carta de respuesta a José María de Cossío, "la región no es una unidad folklórica" (cf. Fraile Gil, 2009, 80); claro que cabría añadir que no lo es ni más ni menos que la provincia o la nación. Las "unidades" que -con mayor motivo- podrían relacionarse con lo que en 
Antropología se denominó, hace ya tiempo, "áreas culturales" serían, en los casos y contextos a los que estamos aludiendo, las comarcas o zonas de donde proceden muchas de las versiones que han nutrido, durante años, los romanceros provinciales y regionales. Es decir, un conjunto o ecosistema geográfico, económico y cultural, que -más allá de la mera correlación entre áreas ecológicas y rasgos culturales- explicaria históricamente por qué se producen las diferencias que conocemos entre los grupos humanos de unas zonas y otras. Pues, como estableciera Daryl Forde, "empíricamente el mundo puede ser dividido en un cierto número de áreas culturales, en las cuales ciertas técnicas, economías y formas sociales dominan la actividad humana", pero aunque las condiciones medioambientales operen como un factor limitador nunca llegarán a ser totalmente determinantes de las posibles combinaciones de la variabilidad cultural (Forde, 1966, 486).

¿Qué "unidades folklóricas" resultarian entonces válidas?, ¿las marcadas por la difusión de los tipos de versiones de un romance o un cuento? ¿No será más bien que esos tipos de versión responden a la diversidad de áreas culturales de que proceden y en las cuales se siguen difundiendo? Porque, si bien se ha dicho y reconocido - por los mismos que luego abogarían a favor de lo opuesto- que las colecciones regionales e incluso provinciales ofrecen algunas "evidentes ventajas prácticas" para la recopilación y estudio de romances (Cid, 1997, III), no es menos verdad que la actividad de estas recolectas, casi siempre llevadas a cabo por folkloristas y eruditos locales, a menudo ha sido mirada con desconfianza, si no con verdadero rechazo, por parte de quienes, pretendiendo trabajar a una escala nacional e incluso supra-nacional (aunque hispánica), se consideraban a sí mismos los únicos "profesionales" o expertos capacitados para desarrollar esa labor. Y, sin embargo, la utilidad de estas recopilaciones modestas, realizadas frecuentemente en áreas que esos otros investigadores muchas veces no pisaron o tardaron en visitar, no reside sólo en que fueran efectuadas por recolectores que conocían mejor a la zona y sus habitantes, o por el efecto de aprecio y valoración del patrimonio propio que esas búsquedas promovidas por "nativos" habrian de provocar en sus paisanos.

Además de todo ello, que no deja de ser -en lo fundamental- bastante cierto, hay a menudo una "toma de pulso etnográfico" (por así llamarlo) de los lugares y sus pobladores que no suele producirse en las mego-encuestas, en los grandes catálogos, en las obras pretendidamente enciclopédicas. Por una razón muy simple, porque este etnógrafo de a pie sí que -siempre- estuvo y se demoró allí, sí llegó a preguntarse y -por lo tanto- a averiguar (en cierta medida) de dónde venían los "productos" que estaba recogiendo, por qué seguían cayendo frutos del tantas veces elogiado "árbol del romancero" o "de la tradición" y de qué modo su "alada semilla" se había transformado al saltar de la Península a América (Menéndez Pidal, 1966, 27).

Ulpiano Leda Ferreras recoge otros pareceres, formulados desde las antípodas de ese benévolo y acogedor ámbito del romancero como "casa común", en que se daba la bienvenida con alborozo a los trabajos provinciales y regionales aportados por los distintos "trabajadores de campo". Señala este autor opiniones muy diferentes a las que hemos venido leyendo hasta ahora, como las de Diego Catálan desde finales de los ochenta a propósito del "peligro de una excesiva proliferación de publicaciones" u otras acerca de que la publicación del material romancístico habría de ser selectiva y teniendo siempre en el horizonte la "tierra prometida" de la edición del Romancero tradicional de las lenguas hispánicas, criticándose "el apoyo que las instituciones provinciales y autonómicas conceden a la publicación de romances regionales por su 'cuestionable utilidad científica'" (Lada Ferreras, 1993, 365).

Dice Ulpiano Lada Ferreras a este respecto que "a las opiniones formuladas por Diego Catalán y Jesús Antonio Cid relativas a la orientación que deben seguir los estudios sobre el romancero cabría hacerles algunas matizaciones" que él puntualiza y resume del siguiente modo (Lada Farreras, 1993, 366):

La realidad se impone siempre por encima de los supuestos teóricos ideales y con ella hay que contar. Y es la realidad de los hechos que la vasta obra concebida por Menéndez Pidal y a la que Catalán y Cid consideran el objetivo final de la colección y publicación de textos romancísticos, avanza con suma lentitud y dificultad (...) Frente a esta situación cabe oponer la mayor agilidad y dinamismo que podrian proporcionar las colecciones regionales, llevadas por supuesto a cabo con los más estrictos criterios de cientificidad, organizadas sistemáticamente dentro de una perspectiva pan-hispánica y aprovechando a la vez las ayudas de las instituciones culturales provinciales y autonómicas. Es posible que por esta vía inductiva y descentralizadora se 
genere la publicación de colecciones rigurosas avanzando decididamente en el conocimiento del Romancero en lugar de fomentar la acumulación de datos en archivos de más que dudosa operatividad (Lada Ferreras, 1993, 366).

Añadiendo matizaciones a las matizaciones, podría discutirse tanto el concepto de pan-hispánico, que -según luego iremos viendo- tiene importantes connotaciones, como el de esos supuestos criterios de "cientificidad" en la recopilación del Romancero. En lo que se refiere al primer aspecto, sólo decir por ahora que el concepto de "pan-hispánico" parte de una aproximación nacional a una cultura para extenderla a todas las otras culturas impregnadas por aquélla. En suma, planea sobre tal concepción la sombra de aquellas visiones en que ciertas culturas habrian desempeñado un papel civilizador sobre otras. Es decir, que habría culturas "de primera" y "de segunda", grandes y pequeñas culturas, reminiscencias primitivas y excelsas civilizaciones que, por el hecho de serlo, tendrían derecho a todo.

0 ¿por qué si no llamar pan-hispánicos a los vestigios de un género baladístico como el romancero en los lugares del mundo donde hubo presencia de la nación española? Por otro lado, el segundo aspecto -relativo a la "cientificidad"- podría llevarnos muy lejos del asunto que constituye el principal objeto de este trabajo; únicamente apuntar que si el "estatus científico" de las recopilaciones lo dicta qué se recoge más que el "cómo", habría que aclarar antes por qué nada más fue "científico" recoger durante mucho tiempo un determinado tipo de romances (los tenidos por "tradicionales") y no toda balada en español, desaprovechándose así la posibilidad de haber recopilado muchas más versiones orales del romancero denominado "vulgar".

Y ya que menciono este término, cómo no reflexionar sobre esa gradación de excelencias romancísticas, desde el romance tradicional y viejo -situado habitualmente en lo más alto de la escala compositiva dentro de los estudios sobre romancero- al romance de ciego, vulgar y contemporáneo (relegado, por el contrario, al lugar más bajo y desdeñable). Este enfoque no sólo dificultaría durante bastante tiempo una cabal visión de conjunto de la baladística y la tradición oral españolas (o, si se prefiere, hispanas), sino que también condicionaría el carácter de los materiales recopilados hasta el punto -en algunos casos- de desfigurar la realidad de las expresiones culturales que se pretendía documentar. Por ejemplo, en lo que respecta a la persistencia, oralización y tradicionalidad de esos romances de ciego tan despreciados, cuya interinfluencia con otras clases de composiciones romancísticas de procedencia más antigua también era sistemáticamente negada.

Esa distinción no sólo entre clases de balada -pues el romancero constituye la mayor parte de la baladística en castellano, pero no toda-, sino por su valor intrínse$c 0$, resultando unas dignas de ser recogidas, archivadas $y$ estudiadas, y otras no, parece estar apuntando hacia algo más que una neutra y aséptica forma de catalogar. Sobre todo, si tenemos en cuenta que quienes se yerguen en mediadores de lo popular suelen acabar constituyéndose en garantes, autentificadores y, en definitiva, "guardianes de la tradición"; pues es este guardián el que "decide quién puede ser o no portador de material 'autentificable' y qué material lo es" o "garantiza que el trasvase de lo oral a lo escrito se hace de la forma adecuada" (Díaz Viana, 1999, 79). Por lo que unos criterios que se nos presentan como "científicos", pero que -sobre todo- lo que hacen es contribuir a la imposición de un canon "estético" ( $y$, en consecuencia, subjetivo) terminan afectando directamente a la realidad: "Los colectores de cantos populares no nos cuentan cómo es lo que existe o cómo ellos lo ven, sino que en su práctica selectiva de rescatar lo que juzgan valioso -dentro del conjunto de una cultura- deciden lo que 'es', qué existe y qué no. Los recopiladores 'autorizan' lo que debe existir" (Díaz Viana, 1999, 80).

Hay, sin duda, una idea -y una ideología- sobre el pueblo y la nación (o el pueblo-nación) detrás de estas clasificaciones y pretensiones de "cientificidad". Y esa noción resulta fácilmente identificable con las visiones de un pueblo eterno, sujeto de la historia y que conservaría las esencias de la nación, un pueblo ya vislumbrado por Herder -y consagrado en general por el Romanticismo- que tendría en cada caso "un carácter único, que se manifestaba en todas sus costumbres e instituciones, sus obras de arte y literatura, y que era intrínsecamente valioso por sí mismo" (Shenk, 1983, 41). A ello volveremos luego, pero lo que nos interesa ahora confirmar es la extraordinaria coherencia que -por paradójico que pueda parecer- esa concepción de pueblo tiene con la estrategia "anti-regionalista" (por así llamarla) en el caso que nos ocupa.

Aunque a veces la búsqueda del originario "pueblo" al que se pertenece produce la multiplicación del virus román- 
tico de los nacionalismos hasta el infinito, y ese impulso favorece regionalismos y localismos de todo tipo, lo cierto es que el escamoteo, evaporación o incluso licuación del "pueblo" en la escala que queramos elegir ofrece bastantes ventajas a la manipulación política. Quiere decirse que el pueblo concreto, ya sea la población de un villorrio o los habitantes contantes y sonantes de una comarca, provincia o región, pueden llegar a resultar incómodos y hasta fastidiosos si lo que se pretende instituir es un constructo abstracto de pueblo, nacional e incluso supra-nacional (o sea, "pan-hispánico"); que lo será, entre otras cosas, porque se supone que todo ese pueblo diseminado por el mundo -y a menudo hispánico a su pesar- sabe y canta los mismos antiquísimos poemas, el viejo romancero.

Ideológicamente hablando, pero no es éste el lugar ni el momento para dilucidarlo, el rescate y estudio del romancero se nos presenta, desde este punto de vista, tanto o más que en cuanto a proyecto filológico y científico como un verdadero proyecto nacional, a caballo entre el rescoldo del tradicionalismo español menos montaraz y el aún tonificante regeneracionismo. No es diferente en ello de la mayoria de las corrientes de folklorismo romántico que, en distintos países europeos, propugnaron la recuperación como "monumentos patrimoniales" de la antigua poesía popular.

La oposición de quienes habían declarado promover el salvamento y conservación del legado romancístico a que proliferen recopilaciones regionales y provinciales resulta -de entrada- bastante contradictoria; y no sólo podría interpretarse como un cierto afán o estrategia de monopolización del romancero, sino que -además- plantea una cuestión de mayor calado: a quién o quiénes "pertenece" esa herencia cultural de indudable valor y cuál sería la manera más adecuada de (como suele decirse hoy en día) "gestionarla" patrimonialmente. Es decir, pensando en que favorezca o sirva de algún modo -como tesoro patrimonial que es- a quienes han tenido el mérito de conservarlo y transmitirlo. $Y$ éste es un asunto que, por supuesto, no afecta sólo al romancero, sino a todo patrimonio cultural -sea tangible o intangible-. Piénsese, por poner un ejemplo bien conocido de todos, en aquellas políticas -ya en parte superadas- que propugnaban, para la mejor conservación, estudio y difusión de los hallazgos arqueológicos su traslado a los museos arqueológicos nacionales bajo la vigilancia y control de los especialistas correspondientes. Desde hace ya tiempo, viene prefiriéndose dentro de ese campo favorecer la observación in situ de los respectivos yacimientos y la exhibición en salas o museos cercanos de las piezas más emblemáticas -siempre que sea técnicamente posible garantizar su integridad y óptimo estado de conservación-. Así se cree -y parece bastante plausible tal criterio- que se "devuelve" de algún modo a la localidad de donde procede ese patrimonio, lo que se habría obtenido previamente de ella, pues se revitalizaría la zona mediante la atracción sobre su singularidad cultural, consiguiéndose de este modo el inmediato aumento de número de visitantes que los restos arqueológicos suelen comportar.

Con el tiempo, la opinión que ha ido ganando terreno en los distintos temas relacionados con la política cultural -tanto material como inmaterial- al respecto es que, justamente, la gestión local y descentralizada de los diversos patrimonios es más dinámica y sostenible que las que antaño solían proponerse. Ulpiano Lada resume -como ya hemos visto- en "agilidad y dinamismo" (Lada Ferreras, 1995, 366) las bondades que ofrecerían las colecciones regionales de romances frente a otras soluciones y el propio Cid reconocia, entre las ventajas de las mismas, el hecho de que -en esa manera- "los lectores tienen motivos adicionales para valorar la obra como algo más 'propio' y enraizado en su ámbito histórico y su hábitat cultural" (Cid, 1997, 3). $Y$ es que, por volver al símil arqueológico, y a pesar de lo paradójico que pueda parecer, adquiere generalmente más repercusión, visibilidad, funcionalidad y sentido un mosaico romano conservado en el lugar donde fue encontrado que extraído de su contexto y llevado a una gran sala de un museo nacional donde quedará anegado y semioculto entre una decena de mosaicos parecidos.

Pues, volviendo al romancero y a esa supuesta incompatibilidad entre un coleccionismo regional y otro nacional e incluso pan-hispánico, ¿por qué ha de aceptarse que la de nación es mejor escala que otras? Tratándose de etnografías $-y$ al fin y al cabo es del trabajo de campo etnográfico de lo que estamos hablando- esa suposición resulta más que discutible. $Y$, sobre todo, ¿por qué sería más "científico" trabajar en un plano nacional que en otro de menor tamaño? ¿Por qué el estatus de cientificidad estaría a salvo de toda sospecha si el enfoque de recopilación y edición de romances se plantea desde la escala de nación y ha de ser cuestionado a priori si se manejan otras escalas o modelos? 
Se ha quedado -también- algo anticuada esa retórica que en tiempos se utilizó para enfatizar que el romancero era expresión cabal de lo español, de lo hispánico o pan-hispánico, cuando -en realidad- lo que se acostumbra a valorar ahora es que la pervivencia oral de los romances constituye algo más interesante, una manera de narrar -cantando- unos imaginarios mucho más locales que nacionales. Y de ahí las variadas soluciones -también éticas- que las distintas versiones dan a los mismos problemas $-0 \mathrm{mi}-$ tologemas- que en los temas romancísticos se plantean. El factor eufemístico (tan importante en los desenlaces de los romances) que, hace ya años, Nascimento señaló oportunamente -sin que por ello tales aspectos Ilegaran a ser suficientemente tenidos en cuenta por otros recopiladores y estudiosos después- explicaría, por ejemplo, desde la diversidad local, que las versiones romancísticas suelan diferir más en los fragmentos finales que en otros; así en lo que atañe a la desaparición o transformaciones del pasaje en que se produce la aceptación del incesto por parte de Delgadina (Nascimento, 1972, 233-275).

Porque si lo que se busca es "poner en valor" -como actualmente se dice- el patrimonio cultural de un territorio nada mejor que contar con recopiladores avezados, con etnógrafos ya curtidos en la labor de campo, que sepan llegar al saber de las gentes desde su conocimiento del contexto en que ese aparente "milagro" de la creación y transmisión populares se produce. Gracias a toda la información que estos compiladores proporcionarán, se podrá documentar con mayor detalle cómo algunos romances han pervivido por una funcionalidad adquirida con el tiempo, que los convirtió en parte o elemento insoslayable de determinadas tradiciones y rituales, como es el caso de los que se siguieron cantando con fines petitorios en Aguinaldos y Marzas o -a modo de música ancestral- en esos grandes bailes identitarios que son las danzas romancísticas. El recopilador, si es etnógrafo o antropólogo tenaz y no encuestador apresurado por un medio rural que -a menudo- desconoce casi por completo, no sólo contextualizará la pervivencia ritualizada del romancero; también describirá -a través de las manifestaciones de sus informantes- cómo se han transmitido intergeneracionalmente estos cantos: de padres a hijos $y_{\text {, }}$ en algunos casos, de abuelos a nietos. 0 , simplemente, de viejos a jóvenes, y -frecuentemente- de viejas a mozas, cuando unas y otras coincidian en tareas como las de coser o cuidar las vacas.
La cuestión de fondo reside en qué es lo que nos parece realmente importante: ¿Acumular cientos y cientos de versiones de un tema, convenientemente descontextualizadas para que su comparación sea más fácil, o averiguar todo lo que esté a nuestro alcance sobre cómo se ha producido y produce el proceso de creación y transmisión de un género tan destacado dentro de la literatura popular como es el del romancero? Pues podría decirse, aquí también, que una y otra cosa no son ni deberían ser necesariamente incompatibles -lo que es cierto-, pero en la práctica la teoría y método utilizados para conseguir esos propósitos han llegado a ser lo suficientemente distintos como para que, desde la primera de estas perspectivas al menos, se haga muy arduo conjugar la mera acumulación de textos parecidos y comparables con la indagación sobre la creatividad colectiva que los hace posibles.

Se tiende inevitablemente a desvirtuar, en la primera forma de trabajar, lo característico de cada versión, lo que la hace única, no sólo por la incidencia que las creencias morales de la colectividad puedan tener -por ejemplo- en el desenlace de los romances que se ocupan del incesto, sino también por la abstracción a la que se somete a una realidad mucha más compleja de lo que, al final, parece. Uno de los aspectos tampoco casualmente desdeñados y casi desaparecidos de buena parte de la bibliografía de recopilaciones romancísticas, a lo largo de su ya extensa historia, es el de la música. Lo que obvia u omite -sin duda- la importancia crucial que la melodía siempre ha tenido para la propia transmisión y pervivencia del género, pero -además- viene a soslayar de nuevo un sinfín de connotaciones "molestas": la manera de interpretar, el acompañamiento musical propio de cada lugar, el modo de colocar la voz... En suma, deja en el anonimato las voces, que es como decir las gentes. Pero incluso propicia la desaparición de todo ese contexto cultural del que cada música resulta ser al tiempo argamasa, sintesis y conjunción singular.

Porque las melodias contextualizan y contribuyen a localizar al romancero, a enraizarlo en un lugar determinado. Curiosamente, las colecciones regionales suelen atender a esta idiosincrasia musical mucho más que las publicaciones de enfoque nacional o supranacional. El plan de documentar todas o -más bien- la mayor parte de las versiones del romancero pan-hispánico en su texto y melodías no es que tenga aún lejos sus horizontes, es que éstos siempre lo 
estarán; ya que pensar que se trata de una obra abarcable es ver al romancero como un cuerpo que se mantuviera estático. Pero el romancero, como el pueblo que lo sustenta y lo difunde, no es un corpus inmóvil: la realidad se mueve. No es fácil, sino más bien imposible o ilusorio, conseguir una foto fija y totalizadora del romancero oral. Intentar comprimir en una sucesión que parece -y lo es- interminable de tomos la descripción absoluta del estado de los romances populares raya con lo quimérico.

No es nuestro propósito aquí entrar en la discusión sobre las distintas acepciones que pueden darse $-y$ de hecho se han dado- del concepto de pueblo, ni menos ocuparnos de desentrañar las interpretaciones del término "literatura popular" con el que es habitual referirse al romancero. El lector podrá encontrar en algunos trabajos anteriores tanto nuestra posición como un resumen de las diferentes aproximaciones y usos que se han venido empleando de vocablos como el de "pueblo" o "lo popular" (Díaz Viana, 1995, 17-21).

Nos interesa, sin embargo, resaltar cómo buena parte de los estudios sobre los romances han estado condicionados en su teoría y método por una visión determinada de pueblo que, en realidad, es más una estrategia para que éste se evapore o diluya en el concepto de "nación" y de lo "nacional" que un verdadero interés por conocer sus expresiones.

Se trata de un auténtico proceso de licuación o transustanciación del pueblo, al que se disuelve como un azucarillo de identidad local en otra nacional o supranacional a su paso -por ejemplo- a través de los alambiques que permiten extraer del romancero una síntesis o esencia de lo español e hispánico. Pero tal licuación es también la liquidación del pueblo en cuanto folk o conjunto de personas identificable y reconocible. Pues ¿qué se gana y qué se pierde al cabo con ese sistema de trabajo que extrae versiones y variantes en número indefinido del saber popular ignorando por completo las circunstancias que explicarían su pervivencia? Se gana la facilidad de comparación al convertir performances musicales muy dispares en textos homogeneizados y comparables: se pierde, como ya se ha señalado, nada menos que al pueblo, o dicho de manera menos romántica, la realidad de unas gentes o folks que se transmiten su saber local. Y la pregunta sustancial, respecto a todo lo que venimos tratando, es si no resultará -entonces- preferible estudiar el conocimiento local también localmente. Además de, por supuesto, reivindicarlo como objeto antropológico de estudio.

Porque, aunque podamos aceptar que el pueblo finalmente "somos todos" (Dundes, 1977, 21), habrá que precisar que unos somos más pueblo que otros. Es cierto, como señalara Burke, que las élites también participaban de la cultura popular y la conocian tan bien o mejor que el vulgo en la Europa moderna (Burke, 1981, 3-22), pero no lo es menos que habia clases populares que no participaban de eso que todavía algunos siguen Ilamando "gran" o "alta cultura", la de las clases dominantes, la hegemónica.

Las cosas parecen estar cambiando, pero -durante mucho tiempo- al pueblo en cuanto grupo de gente, al folk, le caracterizó su enraizamiento en un territorio que conocía e interpretaba de forma colectiva. Las élites europeas, sin embargo, por más que apelasen periódicamente a lo regional o nacional para mejor ejercer su control social y económico, siempre tendieron al cosmopolitismo y a despegarse de los lugares de que procedian. ¿Por qué algunos hemos preferido desde antiguo la utilización del término "popular" para referirnos a una literatura que escapaba a la supervisión de las élites? Porque lo "popular" obvia el sentido o significado que se quiera dar a la palabra pueblo y alude a una creación literaria de participación colectiva en proceso donde ni la autoría ni la forma escrita son lo relevante. Más que reivindicar la utilización de un término como el de literatura popular debería explicarse "por qué ha llegado a ser considerada únicamente tal (literatura) -en un ámbito como el occidental- la literatura escrita, resultando todas las demás expresiones del arte de la palabra que quedaban al margen de ella tan relegadas que ha sido preciso justificarlas y darles un nombre diferente" (Díaz Viana, 2007, 30).

Lo tradicional, sin embargo, más allá de describir unas fases de transmisión cultural mediante consenso colectivo que -por otra parte- son comunes a otros procesos de difusión de la cultura en general (pues toda cultura se transmite por consenso y tradición), ha servido precisamente para que las élites pudieran llegar a ejercer un control de aquellas zonas de la cultura que aún no controlaban; sirvió -en definitiva- a manera de filtro respecto a la escala y tipo de pueblo o creación popular que resultaban aceptables 0 asumibles. 
Porque las gentes identificadas con el pueblo o el vulgo durante la Edad Media, pero también en el Renacimiento, eran fundamentalmente aquéllas que no pertenecian a las clases privilegiadas y se veían supeditadas a ellas. Y de ahí que lo "vulgar" tuviera y siga teniendo una connotación negativa, a pesar de que los humanistas españoles identificaran -acertadamente- a lo "vulgar" con el saber común o de todos y buscaran descubrir en él restos de los viejos conocimientos y costumbres del mundo antiguo. Hay ya, entonces, un primer "descubrimiento del pueblo" o de lo popular que va a favorecer -sin duda- la fundación y desarrollo de unas literaturas identificadas como nacionales. Con el segundo "descubrimiento", el del pueblo por parte de los románticos, la tendencia va a cambiar y se va a pasar en general de la visión miserabilista del mismo a su mitificación. Casi todo lo que viene del pueblo será entonces bueno y digno de admiración e imitación porque en él se pensaba que residian las bases de la nación, pero ello -siendo así en términos generales- no estará exento de algunas importantes salvedades: no todo pueblo vale. El pueblo idealizado por el primer Romanticismo alemán será el campesino, mientras que el urbano será identificado con el vulgo degradado y vocinglero de las ciudades, ese "populacho" que -según la expresión de Herder- "nunca canta o versifica, sólo chilla o mutila" (cf. Bendix, 1997, 39-40). Algo que resuena en ciertas apreciaciones pidalianas -y postpidalianas- de un romancero considerado como "vulgar" y un tipo de cantores populares -el de los ciegos que vendian romances- al que Juan Menéndez Pidal ya tildara de "degenerado descendiente por línea directa del juglar" (Juan Menéndez Pidal, [1885] 1986, X).

El pueblo contemplado desde esta perspectiva, además, sólo interesa en la medida que sirve de reproductor de una época arcaica o dorada, de un pasado glorioso, pero no tanto porque cree e innove realmente. De ahí que la versión herderiana de lo popular sea maniquea y manipuladora, mientras que un pensador como Gramsci, que también -años después- va a reflexionar sobre lo popular, verá mucho más allá: una cultura popular que puede ser conservadora y hasta reaccionaria en ciertos aspectos, pero que -al tiempo- puede ser innovadora e incluso subversiva en otros (Gramsci, [1974]1988, 490). El primero centra su foco en un pueblo que le interesa como "pueblo alemán" y el segundo busca -en las manifestaciones folklóricas- al "pueblo a secas", como clase social con recursos culturales propios desde los que poder afirmarse.
De otro lado, y regresando a nuestro tema, es muy comprensible y hasta loable la postura de Menéndez Pidal en su contexto histórico, pretendiendo extraer y abstraer los aspectos más positivos de la cultura popular, así como defender de paso la importancia y bondad de la literatura española y de lo español en el mundo. Pues su planificación académica habría que enmarcarla en un proyecto nacional de regeneracionismo algo tardío -y además- muy ambicioso que va a colocar su particular foco de interés en aspectos tan vitales en la construcción y proyección de una identidad nacional como son el lenguaje, la historia y la literatura popular (épica y épico-lírica). Y según bien señala Portolés, "la epopeya no es un tema poético cualquiera desde el Romanticismo se la considera la manifestación por excelencia del espíritu de un pueblo" y, de ahí, que Menéndez Pidal "evitara cualquier solución de continuidad en la pervivencia de esta tradición" (Portolés, 1986, 29).

Resulta, con todo, un poco sorprendente que el condicionamiento que tales planteamientos han provocado en los métodos, prácticas y modos de catalogación del romancero no haya sido apenas revisado a lo largo del último siglo, ni modificado un plan archivístico de versiones orales que -por su objetivo prácticamente inabarcable- el tiempo ha revelado como imposible de realizar. El impulso que Pidal proporcionó al interés acerca del romancero, sin embargo sí que ha valido la pena. $Y$ los logros obtenidos desde entonces disculpan con creces, de otra parte, que el proyecto se haya continuado sin apenas renovación ni autocrítica hasta hoy. Lo que no resulta tan defendible es que, desde esa posición predominante concedida por el prestigio de la figura de Menéndez Pidal, se lancen anatemas contra otros modos de recopilar y trabajar, descalificando y poniendo veto a procederes menos centralizadores pero -seguramente- más actualizados y dinámicos.

Ya que el tratamiento de lo popular no es desligable de lo político. En realidad, alude indefectiblemente a una cierta idea de clase, aunque sea lo más confortable manipular el concepto de modo que las clases desaparezcan en pro de la nación y lo popular-local o lo popular-concreto se diluyan en manos de las élites. Así, serán éstas las que manejen como quieran y en su beneficio esas expresiones que -por propio desdén o desinterés- habrían llegado a estar fuera del control de su poder. Resulta tan significativa como encomiable, en este sentido, la póstuma voluntad de Diego Catalán de que se siga publicando tras su muerte -en un blog- una 
antología de lo que el rebautizó "Archivo del Romancero de La Cuesta del Zarzal" (formado por el Archivo Pidal/Goyri y Archivo Sonoro del Romancero Débora Catalán), para así "poner en manos de todo el mundo", en un afán que parece tener algo de "devolución" al pueblo del que habría salido, un legado que él mismo consideraba "patrimonio de la humanidad" (lacuestadelzarzal@gmail.com).

Dice Glassie que, para quienes estudiamos a la gente 0 sus expresiones, "humanity is our issue" (la humanidad es nuestro tema), pero "the problem of folklore is class" (el problema del folklore tiene que ver con las clases sociales) (Glassie, 1982, 575). Y no podemos dejar de estar, en lo esencial, de acuerdo con él, aunque -como decíamos- la visión de pueblo-nación tan característica de todos los nacionalismos haga que ese aspecto desparezca, pues todos los integrantes de una nación -aparentemente- se convierten en pueblo. $Y$, de esta manera, el pueblo se esfuma en la nación y la tensión o lucha de clases también parece evaporarse con ello. Porque, como sigue argumentando Glassie: "Are the inhabitants of all economic states people?" (¿son los habitantes de todos los estratos económicos pueblo?). 0, más exactamente, "aquellos otros -muertos, oscuros, pobres- (...) ¿son totalmente humanos?" (Glassie, 1982, 575-576). 0, mejor, ¿son tan humanos o exactamente humanos como nosotros lo somos? Aunque solemos aceptar humanidad y camaradería en la gente del pasado, la de otras etnias o la de otras clases, en el fondo tendemos a mirarlos con recelo, si no con superioridad.

Hay mucho de todo esto, sin duda, en la aproximación que todavía hoy se practica sobre las manifestaciones de lo popular. Y es esta cuestión básica la que conviene revisar y seguramente cambiar para trabajar sobre las creaciones del pueblo -ya se trate de ritos o literatura- de otra manera. Pues, como dice también Glassie: "En nuestra cultura la 'gente primitiva' (como todavía les Ilamamos) sirve como fascinante supervivencia de un estadio anterior de nuestro desarrollo y los campesinos como aquellos seres en que una vez se fosilizó nuestro pasado o quienes ilustran la pintoresca degradación de nuestros logros" (Glassie, 1982, 576).

Así que no cabe duda de que nuestra mirada sobre lo popular está inevitablemente condicionada por prejuicios políticos e ideológicos, y que -como en cualquier aproximación a otras formas de cultura- haya que hacer un esfuerzo de comprensión y acercamiento, un postrer intento para desprendernos de esa soberbia superioridad con que solemos mirar a "lo otro". No basta con recolectar productos de ese pueblo tan a menudo "miserabilizado" o "mitificado" (Grignon y Passeron, 1992) y -con frecuencia- las dos cosas a la vez, como caras complementarias de una misma moneda grabada por la incomprensión y la ignorancia: hay que preocuparse por cómo dicho pueblo crea esos supuestos "frutos" milagrosos que son los romances. Y por toda la riqueza de saberes que hay detrás de ellos. Admitamos además -por último- que, de pertenecer a alguien, los conocimientos populares en general $y$, en este caso, los relatos romancísticos de que tratamos, pertenecen al grupo concreto de gentes que aún los mantienen vivos, más allá de los archivos en donde materialmente hayan podido acabar recalando. $Y$ admiremos a sus creadores, gentes que no "iban de artistas" ni pretendieron nunca serlo, pero que -no obstante- construyeron y conservaron entre todos uno de los edificios más bellos y singulares de nuestra literatura.
Recibido: 16 de agosto de 2010 Aceptado: 15 de enero de 2011

\section{BIBLIOGRAFÍA}

Armistead, Samuel G. (2009): "Prólogo", en José Manuel Fraile, Romancero Tradicional de Cantabria, Santander, Fundación Marcelino Botín, pp. 1113.

Bendix, Regina (1997): In Search of Authenticity. The Formation of Folklore Studies, Madison, The University of Wisconsin Press.
Burke, Peter (1981): Popular Culture in Early Modern Europe, New York, Harper \& Row.

Cid, Jesús Antonio (1997): "El Romancero General de Cantabria. Una 'modest proposal' (que no es la de Jonathan Swift)", El Diario Montañés, Suplemento 11-XII, p. III.

Díaz Viana, Luis (1995): "Concepto de literatura popular y conceptos conexos", Anthropos 166-167: 17-21. 
Díaz Viana, Luis (1999): Los guardianes de la tradición. Ensayos sobre la invención de la cultura popular, Oiartzun, Sendoa Editorial.

Díaz Viana, Luis (2007): "Reflexiones antropológicas sobre el arte de la palabra", Signa. Revista de la Asociación Española de Semiótica 16: 17-33.

Dundes, Alan (1977): "Who Are the Folk?", en William R. Bascom, Frontiers of Folklore, Boulder (Colorado), Westview Press, 17-35.

Forde, C. Daryll (1966): Hábitat, economía y sociedad, Barcelona, Oikos-Tau.

Fraile, José Manuel (2009): Romancero Tradicional de Cantabria, Santander, Fundación Marcelino Botín.

Glassie, Henry (1982): Passing the Time in Ballymenone, Fhiladelphia, University of Pennsylvania Press.

Gramsci, Antonio ([1974] 1988): Antología. Selección, traducción y notas de
Manuel Sacristán, Madrid, Siglo Veintiuno Editores.

Lada Ferreras, Ulpiano (1995): "El romancero en este fin de siglo: el romancero asturiano", en José Caramés Lage, Carmen Escobedo de Tapia, Daniel García Velasco (eds.), El discurso artístico en la encrucijada de fin de siglo, Oviedo, Universidad de Oviedo, 363-373.

Menéndez Pidal, Juan ([1885]1986): Poesía popular. Colección de los viejos romances que se cantan por los asturianos en la Danza prima, Esfoyazas y Filandones. Recogidos directamente de la boca del pueblo, edición de Jesús Antonio Cid, Madrid-Gijón, Seminario Menéndez Pidal-Editorial Gredos-GH Editores.

Menéndez Pidal, Ramón (1966): Castilla. La tradición, el idioma, Madrid, Espasa-Calpe.
Nascimento, Braulio do (1972): "Eufemismo e criaçào poética no romancero tradicional", en Diego Catalán, Samuel G. Armistead y Antonio Sánchez Romeralo (eds.), El romancero en la tradición oral moderna: 1 er $^{\mathrm{e}} \mathrm{Co}-$ loquio Internacional, Madrid, Cátedra Seminario Menéndez Pidal, Universidad Complutense de Madrid-Gredos, 233-275.

Grignon, Claude y Passeron, J. C. (1992): Lo culto y lo popular. Miserabilismo y populismo en sociología y literatura, Madrid, ediciones de la Piqueta.

Portolés, José (1986): Medio siglo de filología española (1896-1952). Positivismo e idealismo, Madrid, Cátedra.

Shenk, H. G. (1983). El espíritu de los románticos europeos, México, Fondo de Cultura Económica. 